\section{A Modified Phase Rule Equation}

IT is well known that the Gibbs Phase Rule as applied to equilibrated macro-systems subject to changes of temperature, pressure and concentration may be written in the form

$$
P+F=C+2 \text {, }
$$

where $P$ is the number of phases, $F$ the number of degrees of freedom and $C$ the number of components. In applying the equation to physical and chemical equilibrium, the number of components $C$ is taken as the minimum number of independently variable constituents in terms of which the equilibrium may be expressed in the form of a chemical equation. The term 'number of components' has thus to bear the brunt of the matter and assume a responsibility not shouldered by the equation itself. In fact, it is frequently necessary to write out a properly balanced chemical equation in order to decide upon the number of components in the system. Moreover, the equation cannot be applied without making allowance for certain restrictions when the phases approach identity, as in critical phenomena, or when one of the substances is kept in a definite weight-ratio to some other substance in the system, as in dissociation phenomena.

In view of these difficulties it is perhaps desirable to state the Phase Rule in terms more readily compre. hended by the ordinary chemist and metallurgist. If we retain the original definitions of phases and degrees of freedom and let $C$ be the total number of chemical constituents (that is, actual chemical substances) present in the system we may write

$$
P+F+R=C+2-r,
$$

where $R$ is the number of reversible chemical reactions occurring in the system, and $r$, the restriction term, is equal to the number of pairs of phases having the same concentration or composition. This equation may then be applied without reference to stoichiometric chemical equations and without recourse to any process of choosing components.

Thus in the thermal dissociation of calcium carbonate :

$$
\mathrm{CaCO}_{3} \rightleftarrows \mathrm{CaO}+\mathrm{CO}_{2},
$$

we have three constituents and one reversible chemical reaction so that

$$
3+F+1=3+2-0 \text {; that is, } F=1 \text {. }
$$

Again, in the dissociation of ammonium chloride

$$
\mathrm{NH}_{4} \mathrm{Cl} \rightleftarrows \mathrm{NH}_{3}+\mathrm{HCl} \text {, }
$$

we have a reversible chemical reaction and also one pair of phases (the solid phase and the gas phase) with identical composition, so that

$$
2+F+1=3+2-1 \text {; that is, } F=1 \text {. }
$$

The equation also leads to a clear differentiation between physical and chemical change in transition processes. We know, for example, that the system

$$
\mathrm{Na}_{2} \mathrm{SO}_{4} \cdot 10 \mathrm{H}_{2} \mathrm{O} \rightleftarrows \mathrm{Na}_{2} \mathrm{SO}_{4}+\text { liquid }
$$

containing three substances (decahydrate, anhydrous salt and water) is invariant (that is, $F=0$ ). Accordingly,

$$
4+0+R=3+2-0 \text { and } R=1 .
$$

The point is thus an incongruent melting point arising from the rupture or formation of chemical linkages.
A similar behaviour is exhibited by an intermetallic compound with a submerged maximum, as, for example,

$$
\mathrm{AuSn}_{\mathbf{4}} \rightleftarrows \mathrm{AuSn}_{\mathbf{2}}+\text { liquid ; }
$$

but in this case the linkages involved are metallic. These transition processes are conveniently termed meritectic reactions (from meros, part ; tectic, melting) to distinguish them from the ordinary peritectic reactions of solid solutions. Applying the equation to a binary system of two solid solutions undergoing peritectic reaction,

we have

$$
\alpha \rightleftarrows \beta+\text { liquid, }
$$

$$
4+0+R=2+2-0 \text {; and } R=0 .
$$

It is clear, therefore, that the peritectic transformation of solid solutions does not involve the rupture of chemical or metallic linkages.

The modified equation, while removing the bugbear of the term component, is also of utility in other directions and in my opinion is much more easy to apply than the customary equation.

University College,

S. T. BOWDEN.

Cardiff.

Jan. 17.

\section{Constitution of Heavy Water}

ON lines similar to the investigation by one of us on ordinary water 1,2, we have studied the Raman band of heavy water $\left(99.6\right.$ per cent $\left.\mathrm{D}_{2} \mathrm{O}\right)$ at different temperatures ranging from $6^{\circ}$ to $100^{\circ} \mathrm{C}$. Within this range, the principal band for this substance is very broad and diffuse, extending in its Raman frequencies from 2,254 to $2,784 \mathrm{~cm}^{-1}$. It shows a structure similar to that of ordinary water, having at $30^{\circ} \mathrm{C}$. three components merging into one another

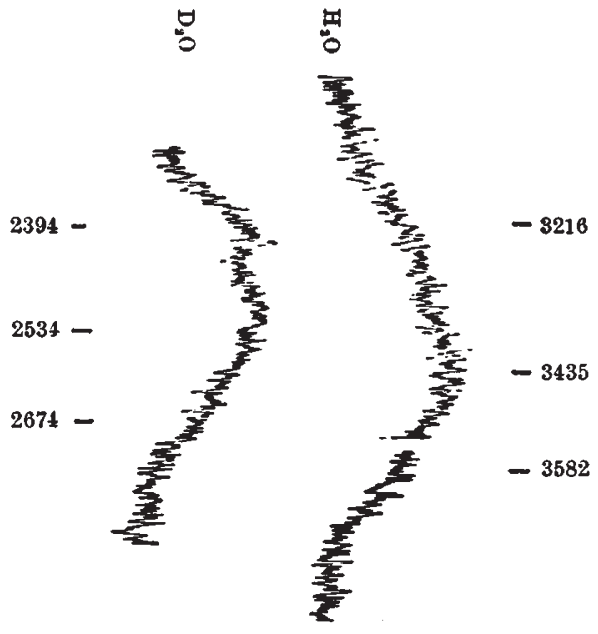

with their maxima at $2,394,2,534$ and $2,674 \mathrm{~cm}^{-1}$. The one important characteristic of this band, as compared to that of ordinary water which earlier workers with this substances, $e^{3,5}$ failed to record, is the difference in the relative intensities of the three components. The accompanying microphotometric curves of the bands for the two types of water were taken at $30^{\circ} \mathrm{C}$. It is clear from the curves that for 